\title{
The finite continuous nonsymmetric Jacobi transform and applications
}

\author{
Fethi Bouzaffour
}

Correspondence: fbouzaffour@ksu. edu.sa

Department of Mathematics, College of Sciences King Saud University, P. O Box 2455 Riyadh 11451, Saudi Arabia

\section{Abstract}

In this paper we consider the differential difference operator

$$
Y^{\alpha, \beta}=-z \frac{d}{d z}+\left\{\frac{2(\alpha+\beta+1)+(\alpha-\beta) z}{1-z^{2}}-(\alpha+\beta+1)\right\} \frac{1-\tau}{2},
$$

where $(\tau f)[z]=f\left[z^{-1}\right]$ The eigenfunction of this operator equal to 1 at 1 is called nonsymmetric Jacobi function. We define the finite continuous nonsymmetric Jacobi transform as an extension of the nonsymmetric Fourier Jacobi series. The basic properties including the inversion formula for this transform are studied. We also derive a sampling expansion associated to $Y^{\alpha, \beta}$.

2010 Mathematics Subject Classification: 33C45, 3352, 94A20.

Keywords: Special functions, Integral transform, Sampling theory.

\section{Introduction}

The differential-difference operators play a prominent role in the theory of special functions and harmonic analysis. Dunkl, Heckman and in particular Cherednik it was next shown that there are related orthogonal systems of special functions which are not Weyl group invariant (see [1-3]), but are in a sense more simple, and from which the earlier Weyl group invariant special functions can be obtained by symmetrization. The specialization of these operators to the case of rank one has its own interest, because everything can be done there in a much more explicit way, and new results for special functions in one variable can be obtained. In the rank one case the Weyl group has order 2, and Weyl group symmetry turns down to a symmetry under the map $t \rightarrow-t$ or $z \rightarrow z^{-1}$ (see [4]).

In this work we consider the differential-difference operator

$$
Y^{\alpha, \beta}=-z \frac{d}{d z}+\left\{\frac{2(\alpha+\beta+1)+(\alpha-\beta) z}{1-z^{2}}-(\alpha+\beta+1)\right\} \frac{1-\tau}{2}
$$

where

$$
(\tau f)[z]=f\left[z^{-1}\right] .
$$


After substitution of $z=e^{-t}, k_{1}=\alpha-\beta, k_{2}=\beta+\frac{1}{2}$, the operator $Y^{\alpha, \beta}$ becomes

$$
\frac{d f(t)}{d t}+\left(k_{1} \frac{1+e^{-t}}{1-e^{-t}}+2 k_{2} \frac{1+e^{-2 t}}{1-e^{-2 t}}\right)(f(t)-(s f)(t))
$$

where

$$
(s f)(t)=f(-t)
$$

Then the operator $Y^{\alpha, \beta}$, coincides with the Heckman's operator [3] (see also [5], $(1,12))$ in the case of root system $B C_{1}$ and the operator $Y^{\alpha,-\frac{1}{2}}$ coincides with Opdam operator corresponding to the root system $A_{1}$.

Moreover, we take $z=e^{-2 i \theta}$, the operator $\frac{1}{2 i} Y^{\alpha, \beta}$ becomes (see [6,7])

$$
\Lambda_{\alpha, \beta} f(\theta)=\frac{d f(\theta)}{d \theta}+[(2 \alpha+1) \cot \theta-(2 \beta+1) \tan \theta] \frac{f(\theta)-(s f)(\theta)}{2} .
$$

The outline of this paper is as follow: In section 2, we show that the unique polynomials eigenfunction of the operator $Y_{\alpha, \beta}$ equal to 1 at 1 is related to Jacobi polynomials and to their derivatives. We call these the nonsymmetric Jacobi polynomials. We establish also a new orthogonality relations for these polynomials and we give integral representation for the nonsymmetric Jacobi function. In section 3, we study the finite continuous nonsymmetric Jacobi transform and we give for this transform an inversion formula. In section 4, sampling theorem associated with the differential-difference operator is investigated.

\section{The nonsymmetric Jacobi function}

\subsection{The nonsymmetric Jacobi polynomials}

We note the Laurent polynomials $f$ in $z$ by $f[z]$. Symmetric Laurent polynomials $f[z]=\sum_{k=-n}^{n} c_{k} z^{k} \quad\left(\right.$ where $\left.c_{k}=c_{-k}\right)$ are related to ordinary polynomials $f(x)$ in $x=\frac{1}{2}\left(z+z^{-1}\right)$ by $f\left(\frac{1}{2}\left(z+z^{-1}\right)\right)=f[z]$. Write

$$
f[z]=f_{1}[z]+\left(z-z^{-1}\right) f_{2}[z]
$$

where

$$
f_{1}[z]=\frac{f[z]+f\left[z^{-1}\right]}{2} \text { and } f_{2}[z]=\frac{f[z]-f\left[z^{-1}\right]}{z-z^{-1}},
$$

are symmetric Laurent polynomials. Consider Jacobi polynomials as normalized symmetric Laurent polynomials:

$$
R_{n}^{\alpha, \beta}[z]=R_{n}^{\alpha, \beta}\left(\frac{1}{2}\left(z+z^{-1}\right)\right)=\frac{n !}{(\alpha+1)_{n}} P_{n}^{(\alpha, \beta)}\left(\left(z+z^{-1}\right) / 2\right)
$$

where $P_{n}^{(\alpha, \beta)}\left(\left(z+z^{-1}\right) / 2\right)$, is the Jacobi polynomials given by (see [8]))

$$
P_{n}^{(\alpha, \beta)}\left(\left(z+z^{-1}\right) / 2\right)=\frac{(\alpha+1)_{n}}{n !}{ }_{2} F_{1}\left(-n, n+\alpha+\beta+1 ; \alpha+1 ; \frac{1}{2}\left(1-\frac{1}{2}\left(z+z^{-1}\right)\right)\right)
$$


For $\alpha, \beta>-1$ these polynomials satisfy the orthogonality relations

$$
<R_{m}^{\alpha, \beta}, R_{n}^{\alpha, \beta}>_{\alpha, \beta}=\int_{0}^{\pi} R_{m}^{\alpha, \beta}(\cos \theta) R_{n}^{\alpha, \beta}(\cos \theta) A^{\alpha, \beta}(\theta) d \theta=h_{n}^{\alpha, \beta} \delta_{m, n}
$$

where

$$
\begin{aligned}
& A_{\alpha, \beta}(\theta)=\sin ^{2 \alpha+1} \theta / 2 \cos ^{2 \beta+1} \theta / 2, \\
& h_{n}^{\alpha, \beta}=\left\{\begin{array}{l}
\frac{\Gamma^{2}(\alpha+1) \Gamma(n+1) \Gamma(n+\beta+1)}{(2 n+\alpha+\beta+1) \Gamma(n+\alpha+1) \Gamma(n+\alpha+\beta+1)}, n=1,2, \ldots, \\
\frac{\Gamma(\alpha+1) \Gamma(\beta+1)}{\Gamma(\alpha+\beta+2)}, n=0 .
\end{array}\right.
\end{aligned}
$$

From the differential equation (2.6) in [9] we deduce that the Jacobi $R_{n}^{\alpha, \beta}[z]$ is the unique solution of the problem

$$
\left\{\begin{array}{l}
\left(L^{\alpha, \beta}\right) f[z]=n(n+\alpha+\beta+1) f[z] \\
f[1]=1 f^{\prime}[1]=0
\end{array}\right.
$$

where

$$
L^{\alpha, \beta}=\left(z \frac{d}{d z}\right)^{2}-\left[\frac{2(\alpha+\beta+1)+(\alpha-\beta) z}{1-z^{2}}-(\alpha+\beta+1)\right]\left(z \frac{d}{d z}\right) .
$$

Theorem 1. For $n \in \mathbb{Z}$ the differential-difference equation

$$
\left\{\begin{array}{l}
\left(Y^{\alpha, \beta}\right) f[z]=\lambda_{n} f[z] \\
f[1]=1
\end{array}\right.
$$

has a unique solution $\mathfrak{R}_{\lambda_{n}}^{(\alpha, \beta)}[z]$ given by

$$
\Re_{\lambda_{n}}^{(\alpha, \beta)}[z]=\left\{\begin{array}{l}
R_{|n|}^{(\alpha, \beta)}[z]-\frac{z}{\lambda_{n}} \frac{d}{d z} R_{|n|}^{(\alpha, \beta)}[z] \text { if } n \neq 0, \\
1 \\
\text { if } n=0 .
\end{array}\right.
$$

where

$$
\lambda_{n}=\lambda_{n}^{(\alpha, \beta)}=\operatorname{sgn}(n) \sqrt{|n|(|n|+\alpha+\beta+1)} .
$$

Proof. From (6), we can write

$$
Y^{\alpha, \beta}=z\left[\left(z-z^{-1}\right) f_{2}\right]^{\prime}+\left[\frac{2(\alpha+\beta+1)+(\alpha-\beta) z}{1-z^{2}}-(\alpha+\beta+1)\right]\left(z-z^{-1}\right) f_{2}-z f_{1}^{\prime} .
$$

Then the following equation

$$
Y^{\alpha, \beta} f[z]=\lambda_{n} f[z]
$$

is equivalent to the system

$$
\left\{\begin{array}{l}
z\left[\left(z-z^{-1}\right) f_{2}\right]^{\prime}+\left[\frac{2(\alpha+\beta+1)+(\alpha-\beta) z}{1-z^{2}}-(\alpha+\beta+1)\right]\left(z-z^{-1}\right) f_{2}=\lambda_{n} f_{1} \\
-z f_{1}^{\prime}=\lambda_{n}\left(z-z^{-1}\right) f_{2} .
\end{array}\right.
$$


Hence,

$$
\left\{\begin{array}{l}
L^{\alpha, \beta} f_{1}=|n|(|n|+\alpha+\beta+1) f_{1} \\
-z f_{1}^{\prime}=\lambda_{n}\left(z-z^{-1}\right) f_{2} .
\end{array}\right.
$$

Then the solution (14) is now immediate.

The Laurent polynomials $\mathfrak{R}_{\lambda_{n}}^{(\alpha, \beta)}[z]$ are called nonsymmetric Jacobi polynomials. For $|z|=1$, we have

$$
\mathfrak{R}_{-\lambda_{n}}^{(\alpha, \beta)}[z]=\mathfrak{R}_{\lambda_{-n}}^{(\alpha, \beta)}[z]=\mathfrak{R}_{\lambda_{n}}^{(\alpha, \beta)}[\bar{z}]=\overline{\mathfrak{R}_{\lambda_{n}}^{(\alpha, \beta)}[z]}
$$

The following formula

$$
z \frac{d}{d z} R_{n}^{\alpha, \beta}[z]=\frac{n(n+\alpha+\beta+1)}{2(\alpha+1)}\left(z-z^{-1}\right) R_{n-1}^{\alpha+1, \beta+1}[z],
$$

leads

$$
\Re_{\lambda_{n}}^{(\alpha, \beta)}[z]=R_{|n|}^{(\alpha, \beta)}[z]-\frac{\lambda_{n}}{2(\alpha+1)}\left(z-z^{-1}\right) R_{|n|-1}^{(\alpha+1, \beta+1)}[z] .
$$

In particular, if $\alpha=\beta=-\frac{1}{2}$, we obtain

$$
\Re_{\lambda_{n}}^{\left(-\frac{1}{2},-\frac{1}{2}\right)}[z]=z^{n} .
$$

\subsection{Orthogonality relations}

Consider $<_{, .} .>_{\alpha, \beta}$, defined in (8), as a symmetric bilinear form on the space of symmetric Laurent polynomials. With the identification $f \leftrightarrow\left(f_{1}, f_{2}\right)$ between a Laurent polynomial $f$ and a pair of symmetric Laurent polynomials (see (6)) we look for a symmetric bilinear form on the space of Laurent polynomials of the form

$$
\left.<g, h>=<g_{1}, g_{2}\right),\left(h_{1}, h_{2}\right)>=<g_{1}, h_{1}>_{\alpha, \beta}+C<g_{2}, h_{2}>_{\alpha+1, \beta+1},
$$

such that the nonsymmetric Jacobi polynomials $\mathfrak{R}_{\lambda_{n}}^{(\alpha, \beta)}(n \in \mathbb{Z})$ given by(16) are orthogonal with respect to this form, i.e.

$$
<\Re_{\lambda_{m}}^{\alpha, \beta}, \Re_{\lambda_{n}}^{\alpha, \beta}>=0(m \neq n) .
$$

By (8) and (16) the orthogonality certainly holds if $|n| \neq|m|$. Thus we have to determine $C$ in (17) such that for $n=1,2, \ldots$,

$$
<\mathfrak{R}_{\lambda_{n}}^{\alpha, \beta}, \mathfrak{R}_{\lambda_{-n}}^{\alpha, \beta}>=0 .
$$

By (17) this turns down to

$$
C=\frac{4(\alpha+1)^{2}}{\lambda_{n}^{2}} \frac{h_{|n|}^{\alpha, \beta}}{h_{|n-1|}^{\alpha+1, \beta+1}}
$$


A priori, it is not clear that $C$ is independent of $n$. However, from(10) we compute

$$
\frac{h_{|n|}^{\alpha, \beta}}{h_{|n-1|}^{\alpha+1, \beta+1}}=\frac{|n|(|n|+\alpha+\beta+1)}{(\alpha+1)^{2}} .
$$

Thus $C$ is independent of $\mathrm{n}$ and the form (17) becomes more explicitly

$$
\left.<g^{\prime} h>=<g_{1}, g_{2}\right),\left(h_{1}, h_{2}\right)>=<g_{1}, h_{1}>_{\alpha, \beta}+4<g_{2}, h_{2}>_{\alpha+1, \beta+1} .
$$

Here $<_{., .}>_{\alpha, \beta}$ is defined by in (8). This form is positive definite if $\alpha>-1$ and $\beta>-1$. The inner product (17) can also be written in terms of an integral with positive weight function. First observe that (6) implies that

$$
f\left[-\left(x \pm i \sqrt{1-x^{2}}\right)^{2}\right]=f_{1}\left(1-2 x^{2}\right) \pm 4 i x \sqrt{1-x^{2}} f_{2}\left(1-2 x^{2}\right) \quad(x \in[-1,1]) .
$$

Hence, for Laurent polynomials $g, h$ we obtain from (17) and (20) that

$$
\begin{aligned}
& <g^{\prime} h>=\int_{-1}^{1} g\left[-\left(x \pm i \sqrt{1-x^{2}}\right)^{2}\right] \overline{h\left[-\left(x \pm i \sqrt{1-x^{2}}\right)^{2}\right]}|x|^{2 \alpha+1}\left(1-x^{2}\right)^{\beta} d x \\
& =\int_{-\pi}^{\pi} g\left(e^{i \theta}\right) \overline{h\left(e^{i \theta}\right)} A_{\alpha, \beta}(|\theta|) d \theta
\end{aligned}
$$

where $A_{\alpha, \beta}(\theta)$, is defined in (9). So the orthogonality of the Laurent polynomials $\Re_{\lambda_{n}}^{\alpha, \beta}$ with respect to the inner product (22) can be rewritten as the following orthogonality for the Laurent polynomials $\mathfrak{R}_{\lambda_{n}}^{\alpha, \beta}$ given by (16):

$$
\int_{-1}^{1} \mathfrak{R}_{\lambda_{n}}^{\alpha, \beta}\left[-\left.\left(x+i{\sqrt{1-x^{2}}}^{2}\right] \overline{\mathfrak{R}_{\lambda_{m}}^{\alpha, \beta}\left[-\left(x+i \sqrt{1-x^{2}}\right)^{2}\right.}|| x\right|^{2 \alpha+1}\left(1-x^{2}\right)^{\beta} d x=0 \quad(m \neq n),\right.
$$

or equivalently,

$$
\int_{-\pi}^{\pi} \mathfrak{R}_{\lambda_{n}}^{\alpha, \beta}\left[e^{i \theta}\right] \overline{\mathfrak{R}_{\lambda_{m}}^{\alpha, \beta}\left[e^{i \theta}\right]} A_{\alpha, \beta}(|\theta|) d \theta=0 \quad(m \neq n) .
$$

\subsection{Extension of the nonsymmetric Jacobi polynomials}

The mapping $n \rightarrow R_{n}^{\alpha, \beta}[z]$, extends to a holomorphic function on $\mathbb{C}$ defined by the same formula with $n$ replaced by $\mu-\rho$ (see [9]). The function $R_{\mu-\rho}^{(\alpha, \beta)}[z]$ is an symmetric entire function in $\mu$, satisfying the relation

$$
R_{\mu-p}^{(\alpha, \beta)}[z]=R_{-\mu-p}^{(\alpha, \beta)}[z], p=\frac{\alpha+\beta+1}{2} .
$$

In the next theorem we give a new integral representation for the Jacobi function.

Theorem 2. For $\alpha>-\frac{1}{2}, \beta>-1$ and $\lambda, \mu \in \mathbb{C}$ such that $\mu^{2}=\lambda^{2}+\rho^{2}$, the function $R_{\mu-\rho}^{(\alpha, \beta)}(\cos \theta)$ has the Laplace integral representation

$$
R_{\mu}^{(\alpha, \beta)}(\cos \theta)=\int_{0}^{\theta} \mathscr{K}(\theta, \phi) \cos (\lambda \phi) d \phi,
$$


with

$$
\mathscr{K}(\theta, \phi)=\mathscr{K}_{o}(\theta, \phi)+\int_{\phi}^{\theta} \mathscr{K}_{o}(\theta, \varphi) \frac{\partial}{\partial \varphi}\left[J_{0}\left(\rho \sqrt{\varphi^{2}-\phi^{2}}\right)\right] d \varphi
$$

and

$$
\begin{aligned}
\mathscr{K}_{0}(\theta, \phi)= & \frac{2^{\alpha-\frac{1}{2}} \Gamma(\alpha+1)}{\frac{1}{2} \Gamma\left(\alpha+\frac{1}{2}\right)}\left(\sin \frac{\theta}{2}\right)^{-2 \alpha}\left(\cos \frac{\theta}{2}\right)^{-\beta-\frac{1}{2}}\left(\cos \frac{\phi}{2}-\cos \frac{\theta}{2}\right)^{\alpha-\frac{1}{2}} \\
& \times{ }_{2} F_{1}\left(\frac{1}{2}+\beta, \frac{1}{2}-\beta ; \alpha+\frac{1}{2} ; \frac{\cos \frac{\theta}{2}-\cos \frac{\phi}{2}}{2 \cos \frac{\theta}{2}}\right) .
\end{aligned}
$$

Proof. We start with the integral representation in [9]

$$
R_{\mu}^{(\alpha, \beta)}(\cos \theta)=\int_{0}^{\theta} \mathscr{K}(\theta, \phi) \cos (\mu \phi) d \phi,
$$

and from [10], we have the integral representation

$$
\cos (\mu \phi)=\cos (\lambda \phi)+\int_{0}^{\phi} \cos (\lambda \varphi) \frac{\partial}{\partial \phi}\left[J_{0}\left(p \sqrt{\phi^{2}-\varphi^{2}}\right)\right] d \varphi
$$

where $J_{0}$ is the Bessel function of first kind and index zero. If we substitute the expression (26) in (27) and a simple calculation show the result.

Lemma 1. The operator $Y^{\alpha, \beta}$ satisfies

$$
Y^{\alpha, \beta} \tau=-\tau Y^{\alpha, \beta},\left[z, Y^{\alpha, \beta}\right]=z+\frac{1}{2}\left\{\beta-\alpha+(\alpha+\beta+1)\left(z+z^{-1}\right)\right\} \tau
$$

and

$$
\left(Y^{\alpha, \beta}\right)^{2}=\left(z \frac{d}{d z}\right)^{2}-\left\{\frac{2 p+(\alpha-\beta) z}{1-z^{2}}-p\right\} z \frac{d}{d z}-z\left\{\frac{2 p+(\alpha-\beta) z}{1-z^{2}}\right\}^{\prime} \frac{1-\tau}{2}
$$

where

$$
[A, B]:=A B-B A .
$$

We can use a similar argument to that used in Theorem 2.1 to show that: For $\lambda, \mu \in$ $\mathbb{C}$, such that $\mu^{2}=\lambda^{2}+\rho^{2}$, the differential-difference problem

$$
\left\{\begin{aligned}
Y^{\alpha, \beta} f[z] & =\lambda f[z] \\
f[1] & =1
\end{aligned}\right.
$$

has a unique solution $\Re_{\lambda}^{(\alpha, \beta)}[z]$ given by

$$
\Re_{\lambda}^{(\alpha, \beta)}[z]=\left\{\begin{array}{l}
R_{\mu}^{(\alpha, \beta)}[z]-\frac{z}{\lambda} \frac{d}{d z} R_{\mu}^{(\alpha, \beta)}[z] \text { if } \lambda \neq 0, \\
1 \quad \text { if } \lambda=0,
\end{array}\right.
$$

we call the function $\Re_{\lambda}^{(\alpha, \beta)}[z]$, the nonsymmetric Jacobi function. For $\lambda=\lambda_{n}^{(\alpha, \beta)}=\operatorname{sgn}(n) \sqrt{|n|(|n|+2 \rho)}$ the nonsymmetric Jacobi function $\Re_{\lambda}^{(\alpha, \beta)}[z]$ reduces 
to the nonsymmetric Jacobi polynomials $\Re_{\lambda_{n}}^{(\alpha, \beta)}[z]$. In the next theorem we give an integral representation for the nonsymmetric Jacobi function.

Theorem 3. For $\alpha>-\frac{1}{2}, \beta>-1$ and $\lambda \in \mathbb{C}$, the function $\mathfrak{R}_{\lambda}^{(\alpha, \beta)}\left[e^{i \theta}\right]$ has the Laplace integral representation

$$
\Re_{\lambda}^{(\alpha, \beta)}\left[e^{i \theta}\right]=\int_{-|\theta|}^{|\theta|} K(\theta, \phi) e^{i \lambda \phi} d \phi
$$

where $K(\theta, \varphi)$ is a function on $(-\pi, \pi)$, continuous on $(-|\theta|,|\theta|)$, supported in $[-|\theta|$, $|\theta|]$ and for $|\varphi|<|\theta|$, we have

$$
K(\theta, \phi)=\frac{1}{2} \mathscr{K}(|\theta|, \phi)-\frac{\operatorname{sgn}(\theta)}{2 A_{\alpha, \beta}(|\theta|)} \frac{\partial}{\partial \phi} \mathscr{K}(\varphi, \phi) A_{\alpha, \beta}(|\varphi|) d \varphi
$$

where $\mathscr{K}(\theta, \phi)$ is given by relation (25).

\section{The finite continuous nonsymmetric Jacobi transform}

The finite continuous nonsymmetric Jacobi transform is defined by [9]

$$
\mathscr{F}^{(\alpha, \beta)} f(\mu)=\int_{0}^{\pi} f(\theta) R_{\mu}^{(\alpha, \beta)}(\cos \theta) A_{\alpha, \beta}(\theta) d \theta, \mu \in \mathbb{C}, \alpha, \beta>-1 .
$$

Inversion formula for the finite continuous nonsymmetric Jacobi transform (1.1) has been studied in many papers (see, $[11,12,9,13])$. In particular, it was inverted in the special Gegenbauer cases $\alpha=\beta=$ integer $\geq 0$ by MacRobert. Butzer, Stens and Wehrens [11] considered the Legendre case and pointed the relationship with sampling theory. Subsequent Walter and Zayed [13] found similar results for values such that $\alpha+\beta$ is a non-negative integer. The interest in this transform was revived by the work of $\mathrm{T}$. H. Koornwinder and G. G. Walter [9], who remove the restriction on $\alpha$ and $\beta$, requiring only that $\alpha>-1$ and $\beta>-1$ In this section we define the finite continuous nonsymmetric Jacobi transform and study some of its basic properties. The idea is to replace the nonsymmetric Jacobi polynomials in the Fourier series by the nonsymmetric Jacobi function. Let $f$ be a function on $(-\pi, \pi)$ such that

$$
\hat{f}^{(\alpha, \beta)}(\lambda)=\int_{-\pi}^{\pi} f(\theta) \overline{\Re_{\lambda}^{(\alpha, \beta)}\left[e^{i \theta}\right]} A_{\alpha, \beta}(|\theta|) d \theta
$$

is well-defined for all $\lambda \in \mathbb{R}$. Then $\hat{f}^{(\alpha, \beta)}(\lambda)$ is called the finite continuous nonsymmetric Jacobi transform of $f$. Recall that each function $f$ defined in $(-\pi, \pi)$ may be decomposed uniquely into the $\operatorname{sum} f=f_{e}+f_{o}$, where the even part $f_{e}$ is defined by $f_{e}(x)$ $=(f(x)+f(-x)) / 2$ and the odd part $f_{o}$ by $f_{o}=(f(x)-f(-x)) / 2$.

Proposition 1. For all $\lambda, \mu \in \mathbb{C}$ such that $\mu^{2}=\lambda^{2}+\rho^{2}$, we have

$$
\begin{aligned}
& \hat{f}^{(\alpha, \beta)}(\lambda)=2 \mathscr{F}^{(\alpha, \beta)}\left(f_{e}\right)(\mu)+2 i \lambda \mathscr{F}^{(\alpha, \beta)}\left(J f_{0}\right)(\mu) \\
& =2 \mathscr{F}^{(\alpha, \beta)}\left(f_{e}\right)(\mu)+\frac{4 i \lambda}{\alpha+1} \mathscr{F}^{(\alpha+1, \beta+1)}\left(\frac{f_{0}}{\sin (.)}\right)(\mu)
\end{aligned}
$$


where the operator $J$ is given by

$$
J f(\theta)=\int_{-\pi}^{\theta} f(\vartheta) d \vartheta
$$

Proof. Let $f=f_{e}+f_{o}$, for $\lambda \in \mathbb{C}$, we have

$$
\hat{f}^{(\alpha, \beta)}(\lambda)=2 \mathcal{F}^{(\alpha, \beta)}\left(f_{e}\right)(\mu)+\frac{2 i}{\lambda} \int_{0}^{\pi} f_{0}(\theta) \frac{d}{d \theta} R_{\mu-p}^{(\alpha, \beta)}(\cos \theta) A_{\alpha, \beta}(\theta) d \theta,
$$

with

$$
\mu^{2}=\lambda^{2}+\rho^{2} .
$$

By integration by parts, we obtain

$$
\begin{aligned}
& \int_{0}^{\pi} f_{0}(\theta) \frac{d}{d \theta} R_{\mu-p}^{(\alpha, \beta)}(\cos \theta) A_{\alpha, \beta}(\theta) d \theta \\
& =-\int_{0}^{\pi} R_{\mu-p}^{(\alpha, \beta)}(\cos \theta)\left(f_{0} A_{\alpha, \beta}\right)^{\prime}(\theta) d \theta \\
& =-\mathscr{F}^{(\alpha, \beta)}\left(\Delta_{\alpha, \beta} J f_{0}\right)(\mu) .
\end{aligned}
$$

We deduce (33) from the fact that

$$
\mathscr{F}^{(\alpha, \beta)}\left(\Delta_{\alpha, \beta} J f_{0}\right)(\mu)=-\lambda^{2} \mathscr{F}^{(\alpha, \beta)}\left(J f_{0}\right)(\mu),
$$

where

$$
\Delta_{\alpha, \beta}(u)(\theta)=\frac{d}{d \theta}\left(A_{\alpha, \beta}(\theta) \frac{d}{d \theta}(u(\theta))\right) .
$$

From the formula (15) we have

$$
A_{\alpha, \beta}(\theta) R_{\mu-p}^{(\alpha, \beta)}(\cos \theta)=\frac{2}{\alpha+1} \frac{d}{d \theta}\left[\frac{A_{\alpha+1, \beta+1}(\theta)}{\sin \theta} R_{\mu-p-1}^{(\alpha+1, \beta+1)}(\cos \theta)\right] .
$$

Hence

$$
\mathscr{F}^{(\alpha, \beta)}\left(\Delta_{\alpha, \beta} J f_{0}\right)(\mu)=-\frac{2}{\alpha+1} \mathscr{F}^{(\alpha+1, \beta+1)}\left(\frac{f_{0}}{\sin (.)}\right)(\mu) .
$$

This establish (34).

In the next theorem we derive an inversion formula for the finite continuous nonsymmetric Jacobi transform.

Theorem 4. Let $f \in C^{2 p}$ with $p>\rho+\frac{3}{2}$, we have

$$
f(\theta)=\int_{-\infty}^{\infty} \hat{f}^{(\alpha, \beta)}(\lambda) \overline{\mathfrak{R}_{\lambda}^{(\beta, \alpha)}\left[e^{i \theta}\right]} \frac{\tilde{h}^{(\alpha, \beta)}\left(\sqrt{\lambda^{2}+p^{2}}\right)}{\sqrt{\lambda^{2}+p^{2}}}|\lambda| d \lambda .
$$

where

$$
\tilde{h}^{(\alpha, \beta)}(\mu)= \begin{cases}\frac{4 \pi \sin (2 \pi \mu)}{\Gamma(\alpha+1) \Gamma(\beta+1) \Gamma(1-\rho+\mu) \Gamma(1-\rho-\mu)} & \text { if } 2 \rho \notin \mathbb{Z}, \text { if } 2 \rho \in \mathbb{N} \\ \frac{2 \mu(\mu-p)_{2 \rho} \cos \pi(\mu-p)}{\mu-\rho} & \text { if } 2 \rho \in \mathbb{N} .\end{cases}
$$


Proof. Let $f=f_{e}+f_{o}$, then we have

$$
\begin{aligned}
& \int_{-\infty}^{\infty} \hat{f}^{(\alpha, \beta)}(\lambda) \overline{\Re_{\lambda}^{(\beta, \alpha)}\left[e^{i \theta}\right]} \tilde{h}^{(\alpha, \beta)}\left(\sqrt{\lambda^{2}+p^{2}}\right) \frac{|\lambda| d \lambda}{2 \sqrt{\lambda^{2}+p^{2}}} \\
& =\int_{-\infty}^{\infty} \mathscr{F}^{(\alpha, \beta)}\left(f_{e}\right)(\mu) R_{\mu-p}^{(\alpha, \beta)}(-\cos \theta) \tilde{h}^{(\alpha, \beta)}(\mu) d \mu \\
& +\frac{d}{d \theta} \int_{-\infty}^{\infty} \mathscr{F}^{(\alpha, \beta)}\left(J f_{0}\right)(\mu) R_{\mu-p}^{(\alpha, \beta)}(-\cos \theta) \tilde{h}^{(\alpha, \beta)}(\mu) d \mu .
\end{aligned}
$$

In the other hand it is easy to see that $f_{e} J f_{o} \in C^{2 p}$ with $p>\rho+\frac{3}{2}$ and by Theorem 4.1 of [9], we get

$$
f_{e}(\theta)=\int_{-\infty}^{\infty} \mathscr{F}^{(\alpha, \beta)}\left(f_{e}\right)(\mu) R_{\mu-p}^{(\alpha, \beta)}(-\cos \theta) \tilde{h}^{(\alpha, \beta)}(\mu) d \mu, 0<\theta<\pi .
$$

and

$$
J f_{0}(\theta)=\int_{-\infty}^{\infty} \mathscr{F}^{(\alpha, \beta)}\left(J f_{0}\right)(\mu) R_{\mu-p}^{(\alpha, \beta)}(-\cos \theta) \tilde{h}^{(\alpha, \beta)}(\mu) d \mu, 0<\theta<\pi .
$$

so that

$$
\int_{-\infty}^{\infty} \hat{f}^{(\alpha, \beta)}(\lambda) \overline{\Re_{\lambda}^{(\beta, \alpha)}\left[e^{i \theta}\right]} \tilde{h}^{(\alpha, \beta)}\left(\sqrt{\lambda^{2}+p^{2}}\right) \frac{|\lambda| d \lambda}{2 \sqrt{\lambda^{2}+\rho^{2}}}=f(\theta) .
$$

\section{Sampling theorem}

The well-known Whittaker-Shannon-Kotelnikov theorem says that if

$$
f(t)=\int_{-\pi}^{\pi} g(x) e^{i t x} d x
$$

and if $g \in L^{2}((-\pi, \pi))$, then

$$
f(t)=\sum_{n=-\infty}^{\infty} f(n) \frac{\sin \pi(t-n)}{\pi(t-n)} .
$$

This theorem has been generalized in various directions (see [11,14-16]). In this section we established a sampling theorem associated to the differential-difference operator $Y^{\alpha, \beta}$. We put

$$
S_{n}^{(\rho)}(\lambda)=\pi^{(\alpha, \beta)}(n) \int_{-\pi}^{\pi} \Re_{\lambda}^{(\alpha, \beta)}\left[e^{i \theta}\right] \overline{\Re_{\lambda_{n}}^{(\alpha, \beta)}\left[e^{i \theta}\right]} A_{\alpha, \beta}(|\theta|) d \theta,
$$

where

$$
\left(\pi^{(\alpha, \beta)}(n)\right)^{-1}=\int_{-\pi}^{\pi}\left|\Re_{\lambda_{n}}^{(\alpha, \beta)}\left[e^{i \theta}\right]\right|^{2} A_{\alpha, \beta}(|\theta|) d \theta .
$$

\section{Lemma 2.}

$$
\begin{aligned}
& S_{n}^{(\rho)}(\lambda)=\lambda\left(\lambda+\lambda_{n}\right) \frac{(2|n|+2 \rho) \Gamma(|n|+2 \rho)}{\pi \Gamma(|n|+1)} \\
& \times \frac{\Gamma(\mu-\rho) \sin \pi(\mu-\rho-|n|)}{\Gamma(\mu+\rho+1)\left(\mu^{2}-(\rho+|n|)^{2}\right)} .
\end{aligned}
$$


Proof. From the relation

$$
\frac{d}{d \theta} R_{\mu-p}^{(\alpha, \beta)}(\cos \theta)=\frac{p^{2}-\mu^{2}}{2(\alpha+1)} \sin (\theta) R_{\mu-p-1}^{(\alpha+1, \beta+1)}(\cos \theta)
$$

and

$$
A_{\alpha+1, \beta+1}(|\theta|)=\frac{1}{4} \sin ^{2}(\theta) A_{\alpha, \beta}(|\theta|)
$$

we have

$$
\begin{aligned}
& \int_{-\pi \lambda}^{\pi} \Re_{\lambda}^{(\alpha, \beta)}\left[e^{i \theta}\right] \overline{\mathfrak{R}_{\lambda_{n}}^{(\beta, \alpha)}\left[e^{i \theta}\right]} A_{\alpha, \beta}(|\theta|) d \theta \\
& =2 \int_{0}^{\pi} R_{\mu-p}^{(\alpha, \beta)}(\cos \theta) R_{|n|}^{(\alpha, \beta)}(\cos \theta) A_{\alpha, \beta}(|\theta|) d \theta+2 \frac{\lambda \lambda_{n}}{(\alpha+1)^{2}} \\
& \times \int_{0}^{\pi} R_{\mu-p-1}^{(\alpha+1, \beta+1)}(\cos \theta) R_{|n|-1}^{(\alpha+1, \beta+1)}(\cos \theta) A_{\alpha+1, \beta+1}(|\theta|) d \theta .
\end{aligned}
$$

and by formula (2.9) in [13]the second member of the last equality becomes

$$
\begin{aligned}
& \frac{2 \Gamma^{2}(\alpha+1) \Gamma(|n|+\beta+1)}{\pi \Gamma(|n|+\alpha+1)} \frac{\Gamma(\mu-p+1) \sin \pi(\mu-p-|n|)}{\Gamma(\mu+p)\left(\mu^{2}-(p+|n|)^{2}\right)}+2 \frac{\lambda \lambda_{n}}{(\alpha+1)^{2}} \\
& \times \frac{2 \Gamma^{2}(\alpha+2) \Gamma(|n|+\beta+1)}{\pi \Gamma(|n|+\alpha+1)} \frac{\Gamma(\mu-p) \sin \pi(\mu-p-|n|)}{\Gamma(\mu+p+1)\left(\mu^{2}-(p+|n|)^{2}\right)}
\end{aligned}
$$

and after simplification we obtain

$$
2\left(1+\lambda^{-1} \lambda_{n}\right) \frac{\Gamma^{2}(\alpha+1) \Gamma(|n|+\beta+1)}{\pi \Gamma(|n|+\alpha+1)} \frac{\Gamma(\mu-p+1) \sin \pi(\mu-p-|n|)}{\Gamma(\mu+p)\left(\mu^{2}-(p+|n|)^{2}\right)} .
$$

We have

$$
S_{n}^{(p)}\left(\lambda_{m}\right)=\delta_{n, m}, n, m \in \mathbb{Z} .
$$

Proposition 2. Let $f L L^{2}\left((-\pi, \pi), A_{\alpha, \beta}(|\theta|)\right)$ and let $f$ be once continuously differentiable on $(-\pi, \pi)$. Then

$$
f(\theta)=\sum_{n=-\infty}^{\infty} \hat{f}^{(\alpha, \beta)}\left(\lambda_{n}\right) \pi^{(\alpha, \beta)}(n) \Re_{\lambda_{n}}^{(\alpha, \beta)}\left[e^{i \theta}\right],-\pi<\theta<\pi,
$$

uniformly on compact subsets of $(-\pi, \pi)$.

Proof. Let $f=f_{e}+f_{o}$. The Proposition 3:1 give the following equality

$$
\begin{aligned}
& \sum_{n=-\infty}^{\infty} \hat{f}^{(\alpha, \beta)}\left(\lambda_{n}\right) \pi^{(\alpha, \beta)}(n) \Re_{\lambda_{n}}^{(\alpha, \beta)}\left[e^{i \theta}\right] \\
& =\sum_{n=0}^{\infty} h_{n}^{(\alpha, \beta)} \mathscr{F}^{(\alpha, \beta)}\left(f_{e}\right)(n) R_{n}^{(\alpha, \beta)}(\cos \theta) \\
& +\sin (\theta) \sum_{n=1}^{\infty} \frac{n(n+2 p)}{(\alpha+1)^{2}} h_{n}^{(\alpha, \beta)} \mathscr{F}^{(\alpha+1, \beta+1)}\left(\frac{f_{0}}{\sin (.)}\right)(n-1) R_{n-1}^{(\alpha+1, \beta+1)}(\cos \theta) .
\end{aligned}
$$


Using the relation

$$
\begin{aligned}
& \frac{(n+1)(n+1+2 p)}{(\alpha+1)^{2}} h_{n+1}^{(\alpha, \beta)}=h_{n}^{(\alpha+1, \beta+1)}, \\
& f_{e}(\theta)=\sum_{n=0}^{\infty} h_{n}^{(\alpha, \beta)} \mathscr{F}^{(\alpha, \beta)}\left(f_{e}\right)(n) R_{n}^{(\alpha, \beta)}(\cos \theta)
\end{aligned}
$$

and

$$
\frac{f_{0}(\theta)}{\sin (\theta)}=\sum_{n=1}^{\infty} \frac{n(n+2 p)}{(\alpha+1)^{2}} h_{n}^{(\alpha, \beta)} \mathscr{F}^{(\alpha+1, \beta+1)}\left(\frac{f_{0}}{\sin (.)}\right)(n-1) R_{n-1}^{(\alpha+1, \beta+1)}(\cos \theta) .
$$

The result follows from Lemma 3.1 in [9].

The following theorem gives a series representation for the finite continuous nonsymmetric Jacobi transform.

Theorem 5. Let $f \in C^{2 p}$, where $2 p>2+\max \left(2 \alpha+1, \rho, \alpha+\frac{1}{2}\right)$. Then

$$
\hat{f}^{(\alpha, \beta)}(\lambda)=\sum_{n=-\infty}^{\infty} \hat{f}^{(\alpha, \beta)}\left(\lambda_{n}\right) S_{n}^{(\rho)}(\lambda)
$$

with absolute convergence, uniform on strips of finite width in $\mathbb{C}$ around $\mathbb{R}$.

If we substitute the expression (30) in (3:2) we obtain

$$
\hat{f}^{(\alpha, \beta)}(\lambda)=\int_{-\pi}^{\pi}(V f)(\theta) e^{-i \lambda \theta} d \theta,
$$

where

$$
(\mathrm{V} f)(\theta)=\int_{\pi \geq|\phi| \geq|\theta|} K(\theta, \phi) f(\phi) A_{\alpha, \beta}(|\phi|) d \phi .
$$

Thus, we can expand the function $\hat{f}^{(\alpha, \beta)}(\lambda)$ with the aid of Whittaker-ShannonKotel'nikov theorem.

\section{Acknowledgements}

This research is supported by NPST Program of King Saud University, project number 10-MAT1293-02.

\section{Competing interests}

The author declares that they have no competing interests.

Received: 27 December 2011 Accepted: 9 May 2012 Published: 9 May 2012

\section{References}

1. Dunkl, CF: Differential-Difference operator associated to reflection groups, Trans. Amer Math Soc. 311(1):167-183 (1989) doi:10.1090/S0002-9947-1989-0951883-8

2. Cherednik, I: Double affine Hecke algebras, Knizhnik-Zamolodchikov equations, and Macdonald's operators, Int. Math Res Not. 9, 171-180 (1992)

3. Heckman, GJ: An elementary approach to the hypergeometric shift operators of Opdam, Invent. Math. 103, 341-350 (1991)

4. Koornwinder, TH, Bouzeffour, F: Nonsymmetric Askey-Wilson polynomials as vector-valued polynomials, Applicable Analysis. 90, 731746 (2010) arXiv:1006.1140v1

5. Heckman, GJ, Dunkl operators: Séminaire Bourbaki, Vol. 1996/97 Astérisque 245, 223-246 (1997). Exp. No. 828, 4

6. Chouchene, F: Harmonic analysis associated with the Jacobi-Dunkl operator on ] - $\pi / 2$, $\pi / 2$ [. J Comput Appl Math. 178, 75-89 (2005). doi:10.1016/j.cam.2004.02.025

7. Chouchene, F, Mili, M, Trimeche, K: Positivity of the intertwining operator and harmonic analysis associated with the Jacobi-Dunkl operator on R. Anal Appl. 1(4):387-412 (2003). doi:10.1142/S0219530503000247 
8. Koekoek, R, Swarttouw, RF: The Askey-scheme of hypergeometric orthogonal polynomials and its q-analogue, Report 98-17, Faculty of Technical Mathematics and Informatics, Delft University of Technology. http://aw.twi.tudelft.nl/ koekoek/askey (1998)

9. Koornwinder, TH, Walter, GG: The finite continuous Jacobi transform and its inverse. J Approx Theory. 60, 83-100 (1990). doi:10.1016/0021-9045(90)90075-2

10. Thyssen, M: Sur certain opérateurs de transmutation particuliers, Mem. Soc Sci Liège. 6, 7-32 (1961)

11. Butzer, PL, Steins, RL, Wehrens, M: The continuous legendre transform, its inverse transform, and applications, Internat. J Math Sci. 3, 47-67 (1980). doi:10.1155/S016117128000004X

12. Deeba, EY, Koh, EL: The continuous Jacobi transform, Internat. J Math and Math Sci. 6, 145-160 (1983)

13. Walter, GG, Zayed, Al: The continuous $(\alpha, \beta)$-Jacobi transform and its inverse when $a+\beta+1$ is a positive integer, Trans. AMS. 304 (1987)

14. Abreu, Luis Daniel, Bouzeffour, Fethi: A Paley-Wiener theorem for the Askey-Wilson function transform, Proc. Amer Math Soc. 138, 2853-2862 (2010). doi:10.1090/50002-9939-10-10327-X

15. Bouzeffour, F: A q-sampling theorem and product formula for continuous q-Jacobi functions, Proc. Amer Math Soc 135, 2131-2139 (2007). MR 2299491 (2008k:33055). doi:10.1090/50002-9939-07-08717-5

16. Bouzeffour, F: A Whittaker-Shannon-Kotel'nikov sampling theorem related to the Askey-Wilson functions. J Nonlinear Math Phys 14(3):367-380 (2007). MR 2350096 (2008h:94031). doi:10.2991/jnmp.2007.14.3.6

doi:10.1186/1687-1847-2012-55

Cite this article as: Bouzaffour: The finite continuous nonsymmetric Jacobi transform and applications. Advances in Difference Equations 2012 2012:55.

\section{Submit your manuscript to a SpringerOpen ${ }^{\circ}$} journal and benefit from:

Convenient online submission

- Rigorous peer review

- Immediate publication on acceptance

- Open access: articles freely available online

- High visibility within the field

- Retaining the copyright to your article

Submit your next manuscript at $\boldsymbol{s p r i n g e r o p e n . c o m ~}$ 